Natan Sian das Neves natan.sian@gmail.com Universidade Federal do Rio de Janeiro - (UFRJ)

\section{Uma introdução aos procedimentos numéricos do método das diferenças finitas: um estudo do comportamento de vigas de fundação na engenharia civil}

\section{An introduction to the numerical procedures of the finite difference method: a study of the behavior of foundation beams in civil engineering}

\section{Una introducción a los procedimientos numéricos del método de las diferencias finitas: un estudio del comportamiento de vigas de cimentación en la ingeniería civil}

\author{
Introduction aux procédures numériques de la méthode des \\ différences finies : étude du comportement des poutres de \\ fondation en génie civil
}

\begin{abstract}
Resumo
O processo de ensino-aprendizado nos cursos de engenharia exige, cada vez mais, do binômio docente-discente uma contextualização prática entre fenômenos contidos em campo e os modelos e técnicas matemáticas. Dessa forma, o vigente artigo objetiva a aplicação do método das diferenças finitas (MDF) como ferramenta pedagógica para solução e interpretação do modelo diferencial de viga sob base elástica, que busca elucidar simplificadamente o comportamento da viga de fundação em construções. A sequência metodológica de análise baseia-se na solução de três vigas, simplesmente apoiadas com modelos de carregamento distintos, nas quais se realizam investigações e extrapolações dos resultados em óticas distintas, transitando entre análises, como do campo de deslocamentos, diagramas de esforços internos, influência dos fatores de rigidez do solo e da viga. Para tanto, as respectivas soluções analíticas de cada caso são tomadas como referência de validação do método numérico. Em síntese, os experimentos numéricos apresentaram uma boa aderência com a solução analítica em relação ao comportamento do erro médio. Por fim, essa técnica, juntamente com o entendimento físico do problema, permite aos discentes uma visão global do fenômeno, permitindo uma amplificação do conhecimento em problemas de magnitude maior.
\end{abstract}

Palavras-chave: Diferenças finitas. Interação Solo-estrutura. Modelo de Winkler. Pedagógico.

\begin{abstract}
The teaching-learning process in engineering courses increasingly requires from the professorstudent binomial a practical contextualization between phenomena contained in the field and mathematical models and techniques. In this way, the current article aims to apply the finite differences method (MDF) as a pedagogical tool to solve and interpret the differential beam model under the elastic base, which seeks to elucidate the behavior of the foundation beam in constructions. The methodological sequence of analysis is based on the solution of three beams, supported simply with different loading models, in which investigations and extrapolations of the results are carried out in different optics, moving between analyzes, such as the displacement field, internal effort diagrams, influence of soil and beam stiffness factors. To do so, the respective analytical solutions of each case take as validation references of the numerical method. In synthesis, the numerical experiments showed good adherence with the analytical solution concerning the average error behavior. Finally, this technique,
\end{abstract}


together with the physical understanding of the problem, allows the students a global view of the phenomenon allowing an amplification in problems of greater magnitude.

Keywords: Finite differences. Soil-structure interaction. Winkler model. Pedagogical.

\section{Resumen}

El proceso de enseñanza-aprendizaje en los cursos de ingeniería exige, cada vez más, del binomio docente-discente una contextualización práctica entre fenómenos contenidos en campo y los modelos y técnicas matemáticas. De este modo, el objetivo de este trabajo es la aplicación del método de las diferencias finitas (MDF) como herramienta pedagógica para resolución e interpretación del modelo diferencial de viga bajo base elástica, que busca aclarar de forma simple el comportamiento de la viga de cimentación en construcciones. La secuencia metodológica de análisis está basada en la solución de tres vigas, simplemente apoyadas con modelos de cargamento distintos, en las cuales se realizan investigaciones y extrapolaciones de los resultados en ópticas distintas, paseando entre análisis, como del campo de desplazamientos, diagramas de esfuerzos internos, influencia de los factores de rigidez del suelo y de la viga. Para tanto, las respectivas soluciones analíticas de cada caso son tomadas como referencia de validación del método numérico. En síntesis, los experimentos numéricos presentan una buena adherencia con la solución analítica en relación al comportamiento del error medio. Por fin, esta técnica, en conjunto con el entendimiento físico del problema, permite a los dicentes una visión global del fenómeno, permitiendo una amplificación del conocimiento en problemas de mayor magnitud.

Palabras-clave: Diferencias finitas. Interacción suelo-estructura. Modelo de Winkler. Pedagógico.

\section{Résumé}

Le processus d'enseignement-apprentissage dans les cours d'ingénierie demande, de plus en plus, au binôme enseignant-élève de faire une contextualisation entre les phénomènes trouvés dans la vie pratique du domaine et les modèles et techniques mathématiques. De cette façon, cet article vise à appliquer la méthode des différences finies (MDF) comme outil pédagogique pour résoudre le modèle différentielle de poutre sous une fondation élastique, qui cherche à expliquer de façon simple le comportement de la poutre de fondation dans les constructions. La séquence méthodologique d'analyse est basée sur la solution de trois poutres simplement appuyées par différents modèles de chargement, dans lesquelles des investigations et des extrapolations des résultats sont effectuées dans différentes optiques: des analyses, telles que le champ de déplacement; les diagrammes d'effort interne, l'influence des facteurs de rigidité du sol et des poutres. Pour cela, les solutions analytiques respectives pour chaque cas sont prises comme référence de validation de la méthode numérique. En résumé, les expériences numériques ont montré une bonne adhérence sur la solution analytique par rapport au comportement de l'erreur moyenne. Enfin, cette technique, associée à la compréhension physique du problème, permet aux étudiants d'avoir une vision globale du phénomène, en montrant une amplification des connaissances sur des problèmes majeurs.

Mots-clés: Différences finies. Interaction sol-structure. Modèle Winkler. Pédagogique.

\section{Introdução}

A engenharia civil é uma seara que contempla uma gama de fenômenos físicos presentes na natureza, que, por sua vez, direcionam um grande volume de pesquisas científicas, como técnicas e inovações tecnológicas em campo. Entre as inúmeras vertentes de estudo, destaca-se a área estrutural, que abrange um amplo contexto 
de aplicação na engenharia, transitando de dimensionamentos de peças estruturais clássicas até análises de elementos que possuem interação com o solo, como estacas, sapatas e vigas de fundação.

Nesse contexto, a concatenação entre elementos estruturais e o solo consiste em uma área que apresenta problemas com graus de complexidades diversos, o que acarreta o grande interesse científico no estudo da interação solo-estrutura. Sendo assim, são necessários modelos matemáticos ou experimentais que traduzam os fenômenos a fim de uma maior compreensão e quantificação das grandezas significativas que influenciam o comportamento físico do sistema (WANG; THAM; CHEUNG, 2005).

Os modelos diferenciais analíticos, em especial, apresentam distintos níveis de verossimilhança com o fenômeno real analisado, o que influencia diretamente na complexidade da obtenção de soluções analíticas, impossibilitando, em muitos casos, soluções diretas. Dessa maneira, os métodos numéricos ganharam espaço na solução desses problemas, destacando-se o método dos elementos finitos, o dos elementos de contorno e o das diferenças finitas (HACHICH et al., 1998).

Todavia a exposição de conceitos relativos à física matemática e métodos numéricos não é uma tarefa fácil de transmitir de forma didática dentro do ciclo de formação dos discentes em um curso de graduação. Além disso, existe a necessidade, ainda, de buscar uma correlação entre os aspectos abstratos da matemática com a sensibilidade física do problema estudado, gerando um grande desafio para os docentes no decorrer do processo de ensino.

Em direção à busca por alternativas pedagógicas, o método das diferenças finitas possibilita uma noção intuitiva e didática sobre conceitos e solução de problemas de engenharia via técnicas aproximadas, fornecendo ao aluno uma visão global dos procedimentos numéricos. Na engenharia, é natural aplicar essa técnica em configurações básicas de sistemas estruturais representativos de fenômenos físicos uma vez que o objetivo seja explorar os detalhes e conceitos do método. Logo, um elemento que exemplifica de forma didática a interação entre solo-estrutura e se enquadra em tal contexto é a viga de fundação (ALVES; REIS, 2008; RIBEIRO, 2005), sendo objeto de estudo e investigação numérico do presente trabalho.

\section{Equação diferencial de governo}

O estudo de viga sob uma base elástica ou usualmente na construção civil, denotada como viga baldrame ou de fundação, é uma análise que envolve diversos fatores, relacionados ao comportamento da viga e do solo. Por esse aspecto, devem-se realizar, a priori, análises simplificadas, a fim de compreender como os efeitos estão se dissipando na interação solo-estrutura.

Nessa linha, objetiva-se usar um modelo de solo que seja simples, tanto conceitualmente quanto matematicamente, e, ao mesmo tempo, fornecer uma boa aproximação para constituir o comportamento preliminar do solo. Considera-se a hipótese de Winkler para representação discreta do solo. Matematicamente, tal modelo é traduzido pela Eq. (1).

$$
p=k_{v} w
$$

\section{Em que:}

$p=$ reação do solo volume gerado $(\mathrm{N} / \mathrm{m})$;

$k_{v}=$ coeficiente de reação vertical $\left(\mathrm{N} / \mathrm{m}^{2}\right)$;

$w=$ deslocamento ou recalque $(\mathrm{m})$.

A Equação (1) estabelece que o solo se comporte como um sistema de molas independentes com resposta linear e elástica. De acordo com essa hipótese, a reação do solo é proporcional ao deslocamento ou recalque (BOWLES, 1974; HETÉNYI, 1971). Sendo que $k_{v}=b k$, em que $k$ é o coeficiente de recalque do solo e $b$ é a espessura da viga em contato com solo. Tal constante de proporcionalidade pode ser determinada por três formas distintas: por ensaios, pelo cálculo do recalque de fundações reais ou pelo uso de tabelas típicas, como posto na Tab. 1 (HACHICH et al., 1998). 
Tabela 1 - Relação entre o coeficiente de recalque e tipos de solos

\begin{tabular}{lr}
\hline Tipo de Solo & $\boldsymbol{k}\left[\mathbf{M N m}^{-3}\right]$ \\
\hline Turfa leve - solo pantanoso & 5 a 10 \\
Areia fina de praia & 10 a 15 \\
Aterro de silte, de areia e cascalho & 10 a 20 \\
Argila molhada & 20 a 30 \\
Argila seca & 60 a 80 \\
Silte compactado com areia e pedra & 80 a 100 \\
Cascalho grosso com pouca areia & 150 a 200 \\
Cascalho grosso com pouca areia compactada & 200 a 250 \\
Turfa leve - solo pantanoso & 5 a 10 \\
\hline
\end{tabular}

Fonte: Adaptado de PAVAN; COSTELLA; GUARNIERI (2014).

Uma representação didática do modelo de Winkler é posta na Figura 1, em que está representada a transição do solo contínuo para o sistema discreto de molas. Observe, ainda, que esse tipo de análise é uma etapa fundamental para o ensino aos discentes, ou seja, para evidenciar como ocorre a simplificação de um problema na prática para um modelo matemático acessível, que fornece uma primeira análise e interpretação do problema. Observe a Fig. 1, na ilustração à esquerda, em que se busca interpretar didaticamente uma fundação rasa de uma construção de uma residência comum associada a um carregamento qualquer. $\mathrm{Na}$ ilustração da direita nota-se que a viga é simplificada para um elemento unidimensional e o solo, representado como um sistema de molas.

Figura 1 - Modelo de viga de fundação.
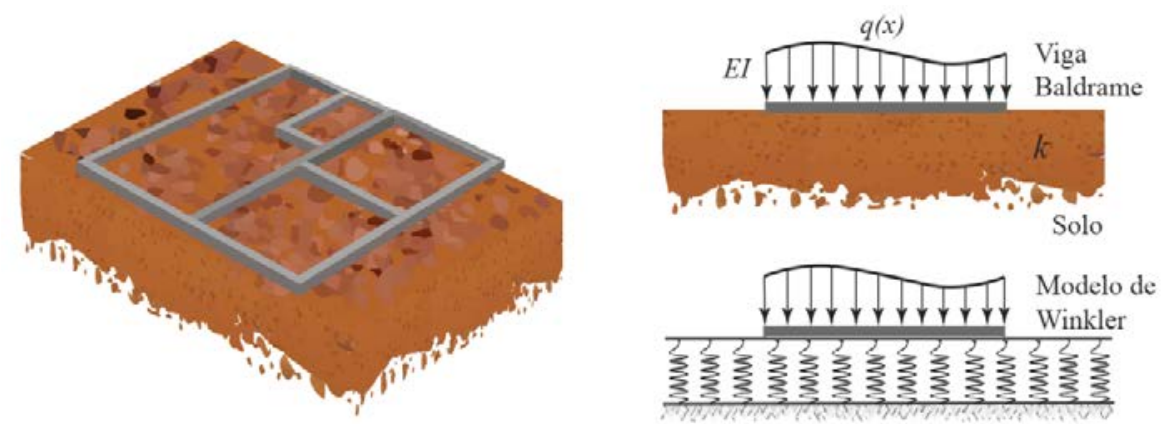

Fonte: Elaborado pelo autor, 2020.

Além disso, o modelo de Winkler apresenta como particularidade que as deformações das molas ocorrem somente na região da fundação em que está sendo aplicado o carregamento, ou seja, não existem deformações das molas nas vizinhanças da estrutura (ANTONIAZZI, 2011; TIMOSHENKO, 1969). Esse modelo busca representar o solo de forma simples, contudo existem modelos mais robustos, que contabilizam outros efeitos como o modelo de Filonenko-Borodich e de Pasternak, vistos com detalhes em Aristizábal-Ochoa, (2003) e Tanahashi (2004).

A equação de governo do problema da viga sob uma base elástica, com base no modelo de Winkler, é constituída a partir de um balanço de forças em um elemento diferencial, como ilustra a Fig. 2. 
Figura 2 - Elemento diferencial da viga.

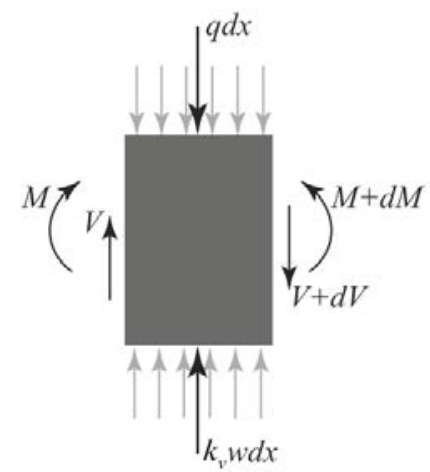

Fonte: Elaborado pelo autor, 2020

Garantindo o equilíbrio das forças no elemento diferencial de viga sob a ação de uma carga distribuída e a reação do solo, tem-se a Eq.(2).

$$
\frac{d V}{d x}=k_{v} w-q
$$

\section{Em que:}

$q=$ carregamento transversal ao eixo da viga $(\mathrm{N} / \mathrm{m}) ;$

$x=$ coordenadas horizontais $(\mathrm{m})$;

$V=$ esforço cortante $(\mathrm{N})$.

Aplicando as relações diferenciais de esforço interno e deformação, pode-se reagrupar a Equação (2) e, assim, apresentar a equação diferencial da viga sob a base elástica respeitando a hipótese de Winkler, conforme expresso pela Eq. (3).

$$
E I \frac{d^{4} w}{d x^{4}}+k_{v} w=q
$$

Em que:

$I=$ momento de inercia da seção $\left(\mathrm{m}^{4}\right)$;

$E=$ módulo de elasticidade do material $\left(\mathrm{N} / \mathrm{m}^{2}\right)$.

Sendo que o produto entre o módulo de elasticidade e o momento de inércia é conhecido como rigidez à flexão da viga. Nota-se que a Equação é similar à equação de Euler para deflexão de viga, a menos de um termo proveniente da reação do solo.

\section{Tratamento numérico do fenômeno}

O método das diferenças finitas é uma técnica numérica que possui como cerne a aproximação das derivadas por expressões algébricas e que relaciona pontos vizinhos contidos no domínio discreto. Usando as expressões de diferenças finitas para aproximar a derivada de quarta ordem do esquema de diferenças centrais, chega-se à Eq. (4) (CUNHA, 2000; JONES, 1997).

$$
\frac{E I}{h^{4}}\left[w_{i+2}-4 w_{i+1}+6 w_{i}-4 w_{i-1}+w_{i-2}\right]+k_{v} w_{i}=q_{i}
$$

Em que:

$h=$ espaçamento entre os pontos da malha numérica $(\mathrm{m})$; 
Dessa forma, reorganizando a Eq. (4), pode-se chegar na equação de diferenças finitas (EDF) para a viga de fundação (NIKODÝM; FRYDRÝŠEK, 2010).

$$
a_{i} w_{i+2}+b_{i} w_{i+1}+c_{i} w_{i}+b_{i} w_{i-1}+a_{i} w_{i-2}=\frac{q_{i}}{E I}
$$

À primeira vista, pode parecer abstrata a ideia de que a Equação (5) é um sistema algébrico, porém note que o índice $i$ varia de 1 até o número de nós na malha, o que introduz um sistema linear. $\mathrm{E}$, ainda, que os coeficientes contidos na Equação (5) são expostos de forma compacta na Eq. (6).

$$
a_{i}=\frac{1}{h^{4}} ; \quad b_{i}=-\frac{4}{h^{4}} ; \quad c_{i}=\frac{6}{h^{4}}+\frac{k_{v}}{E I}
$$

Escrevendo a equação de diferenças finitas para cada nó contido na malha, gera-se um sistema linear, expresso pela Eq. (7).

$$
[K]\{w\}=\{F\}
$$

Em que:

$[K]=$ matriz de rigidez;

$\{W\}=$ vetor resposta;

$\{F\}=$ vetor de carga;

Os elementos contidos na Equação (7) podem ser expandidos na forma matricial, conforme dado pela Equação (8), em que a matriz de coeficiente (ou matriz de rigidez), sendo posta em termos dos coeficientes presentes na Equação, e a vetor de força (ou de termos independentes $F$ ) fica em função da rigidez à flexão e da carga externa distribuída em cada ponto da viga (TEODORU, 2007).

$$
K=\left[\begin{array}{ccccc}
c_{1} & b_{1} & a_{1} & & \\
b_{1} & c_{2} & b_{2} & & \\
a_{1} & b_{2} & & & a_{n} \\
& & & c_{n-1} & b_{n} \\
& & a_{n} & b_{n} & c_{n}
\end{array}\right] \quad w=\left[\begin{array}{c}
w_{1} \\
w_{2} \\
\\
w_{n-1} \\
w_{n}
\end{array}\right] \quad F=\frac{1}{E I}\left[\begin{array}{c}
q_{1} \\
q_{2} \\
\\
q_{n-1} \\
q_{n}
\end{array}\right]
$$

Observe, ainda, que o vetor resposta apresenta o deslocamento em cada ponto da malha, ou seja, se a malha contiver muitos nós, tem-se um mapeamento completo na viga. Note também que a Equação (7) representa o problema global da viga em base elástica, de modo que, para restringir o sistema, devem-se aplicar as condições de contorno envolvidas no problema em questão. Para uma viga biapoiada tem-se que o deslocamento e o momento fletor são nulos nas bordas, como expresso na Eq. (9).

$$
w_{n}=\left.0 \quad \frac{d^{2} w}{d x^{2}}\right|_{n}=0
$$

Sendo que o índice $n$ representa os pontos de extremidade da viga. Logo, aplicando adequadamente a Equação (9) na Equação (7), chega-se ao sistema final, dado pela Eq. (10).

$$
\left[K^{\prime}\right]\{w\}=\left[F^{\prime}\right]
$$

Em que:

[K'] = matriz de rigidez após condições de contorno;

$\left\{F^{\prime}\right\}=$ vetor de carga após condições de contorno; 
O sistema linear expresso pela Equação (10) fica restrito às condições de contorno postas no problema. Destaca-se, ainda, a solidificação de um conceito importante sobre métodos numéricos, isto é, a transição do meio contínuo, que apresenta infinitos pontos, respeitando a equação diferencial para o meio discreto, em que existe um número finito de pontos. Devido essa transição de domínios, introduz-se o erro numérico explicando o motivo dos métodos de solução serem denotados como métodos aproximados.

Figura 3 - Lógica de programação do método das diferenças finitas.

\begin{tabular}{|c|c|c|c|}
\hline \multicolumn{4}{|c|}{ Algoritmo 1: Estrutura Geral do Programa Computacional - Pseudocódigo } \\
\hline \multirow{2}{*}{\multicolumn{2}{|c|}{$\begin{array}{l}1 \text { Pré-Processamento } \\
2 \triangleright \text { Leitura de Dados }\end{array}$}} & \multicolumn{2}{|l|}{24} \\
\hline & & \multicolumn{2}{|c|}{25 ○ Condições de Contorno } \\
\hline 3 & Seção Tranversal: $B, H$ & 26 & $\rightarrow$ Imposição CC : \\
\hline 4 & Comprimento da Viga: $L$ & 27 & $K, F \rightarrow K^{\prime}, F^{\prime}$ \\
\hline 5 & Momento de Inércia: $I$ & \multirow{2}{*}{\multicolumn{2}{|c|}{ א, }} \\
\hline 6 & Módulo de Elasticidade: $E$ & & \\
\hline 7 & Rigidez da Viga: $E I$ & \multicolumn{2}{|c|}{$29 \triangleright$ Solução Sistema Linear } \\
\hline 8 & Coeficiente de Recalque: $k$ & 30 & $w=K^{\prime} F^{\prime}$ \\
\hline 9 & Cargas Externas Aplicadas & 31 & $\rightarrow$ Deslocamentos Nodais \\
\hline 10 & & \multicolumn{2}{|r|}{ 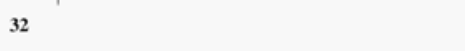 } \\
\hline \multicolumn{2}{|c|}{11 Processamento } & \multicolumn{2}{|c|}{33 Pós-Processamento } \\
\hline \multicolumn{2}{|c|}{$12 \triangleright$ Domínio Discreto } & \multicolumn{2}{|c|}{$34 \triangleright$ Cálculo Secundários } \\
\hline 13 & Número de Pontos: $n_{p}$ & & Rotação: $\theta(x)=w^{\prime}$ \\
\hline 14 & Espaçamento: $h=L /\left(n_{p}-1\right)$ & 36 & Momento Fletor: $M(x)=E I w^{\prime \prime}$ \\
\hline 15 & & 37 & Esforço Cortante: $V(x)=E I w^{\prime \prime \prime}$ \\
\hline \multicolumn{2}{|c|}{$16 \triangleright$ Construção do Sistema Algébrico } & \multicolumn{2}{|c|}{38} \\
\hline 17 & - Matriz Rigidez & \multicolumn{2}{|c|}{ 39 $\triangleright$ Tratamento de Resultados } \\
\hline 18 & $a_{i}=1 /\left(h^{4}\right)$ & 40 & Verificação dos dados \\
\hline 19 & $b_{i}=-4 /\left(h^{4}\right)$ & 41 & Métrica de Erro: \\
\hline 20 & $c_{i}=\left(6 /\left(h^{4}\right)\right)+(k /(E I))$ & 42 & $\varepsilon(\%)=100 \times \frac{\left|u_{\text {ref }}-u_{\text {num }}\right|}{\left|u_{\text {ref }}\right|}$ \\
\hline 21 & $K=f\left(a_{i}, b_{i}, c_{i}\right)$ & 43 & Gráficos: $w, \theta, M$ e $V$ \\
\hline 22 & - Vetor de Força & 44 & Impressão de Dados \\
\hline 23 & $F=f($ Cargas $, h, E I)$ & 45 & \\
\hline
\end{tabular}

Fonte: Elaborado pelo autor, 2020.

Para exemplificar a lógica de programação utilizada na solução do programa de viga sob uma base elástica baseado nos procedimentos numéricos do método das diferenças finitas, a Figura 3 apresenta uma estrutura geral de programação aplicada. Observe que a programação é estrutura em três blocos de código, préprocessamento, processamento e pósprocessamento. $O$ código computacional é desenvolvido em ambiente MATLAB, devido à simplicidade de programação, à produtividade e à disponibilidade de diversos recursos gráficos.

\section{Discussões e testes numéricos}

Para elucidar em exemplos numéricos os conceitos apresentados, analisam-se três casos com carregamentos distintos e a vinculação da viga sendo biapoiada nas extremidades. Nos exemplos numéricos a seguir considera-se o módulo de elasticidade de $E=15 \mathrm{MPa}$. A Figura 4 representa o primeiro caso, que constitui uma viga biapoiada submetida a uma carga distribuída $q=10 \mathrm{kN} / \mathrm{m}$ com comprimento igual a $L=8 \mathrm{~m}$ e seção transversal de $20 \times 15 \mathrm{~cm}$. 
Figura 4: Viga biapoiada com carregamento distribuído ao longo do eixo.

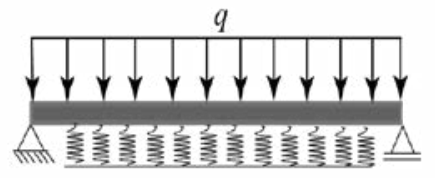

Fonte: Elaborado pelo autor, (2020).

A validação é conduzida pela comparação com a solução analítica, o que se pode verificar com detalhes em Hetényi (1971). A Figura 5 ilustra a curva de erro médio.

Figura 5: Erro médio percentual para viga com carga distribuída.

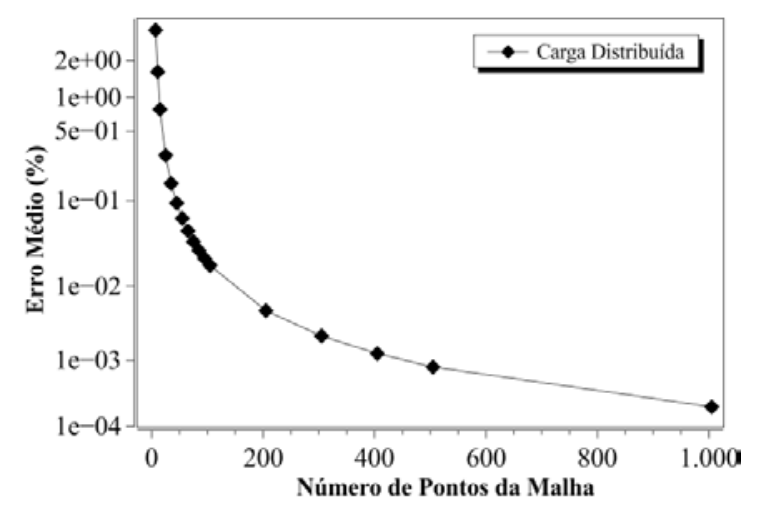

Fonte: Elaborado pelo autor, (2020).

No processo de validação, aplica-se a definição clássica de erro, que é constituída pela razão entre a diferença da solução analítica e numérica e a solução analítica, em módulo. Nota-se que, na Figura 5, o erro tende à nulidade com refinamento da malha. Dessa maneira, analisa-se o comportamento do erro percentual ao longo do comprimento da viga, representa-se na Figura 6 a distribuição do erro.

Figura 6: Distribuição de erro ao longo da viga.

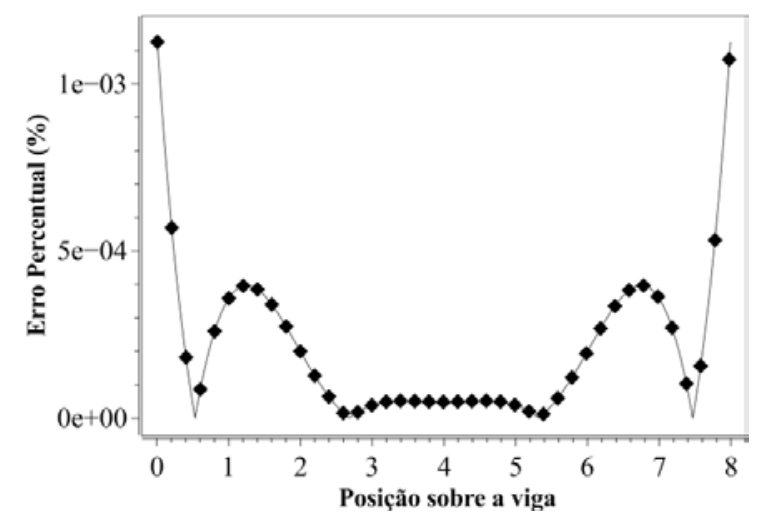

Fonte: Elaborado pelo autor, (2020).

Percebe-se, pela Figura 6, que próximo aos apoios a ordem de grandeza do erro é maior, ao passo que, nas vizinhanças do meio do vão da viga, o erro possui uma ordem de grandeza que apresenta uma menor variação em seu valor. Devido à influência de diversos fatores significativos no problema, é fundamental investigar inicialmente como tais fatores se comportam separadamente. A Figura 7 ilustra o comportamento da viga conforme varia o fator $k$. 
Figura 7: Análise paramétrica de $k$ para campo de deslocamento.

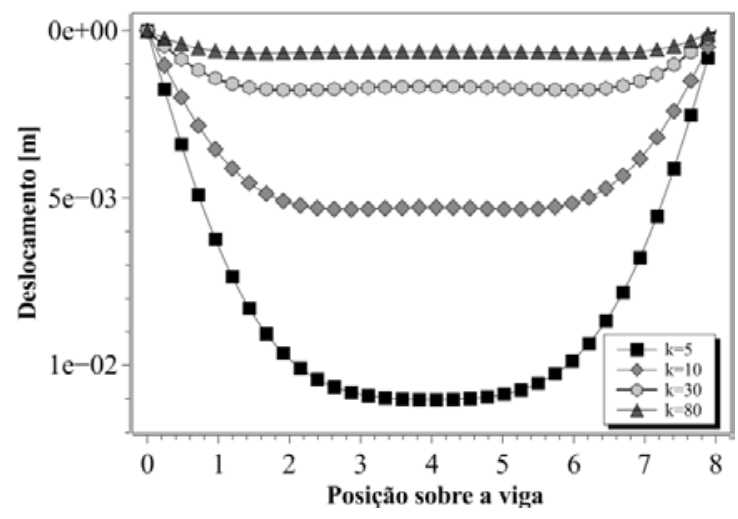

Fonte: Elaborado pelo autor, (2020).

Observa-se que, com o aumento do coeficiente de recalque do solo, o campo de deslocamento transversal da viga tende a um formato de um perfil mais horizontal, devido à influência da reação do solo ao longo da viga. De modo similar, o efeito do solo no comportamento do diagrama de momento fletor da viga é posto na Fig.(8).

Figura 8: Análise paramétrica de $k$ para momento fletor.

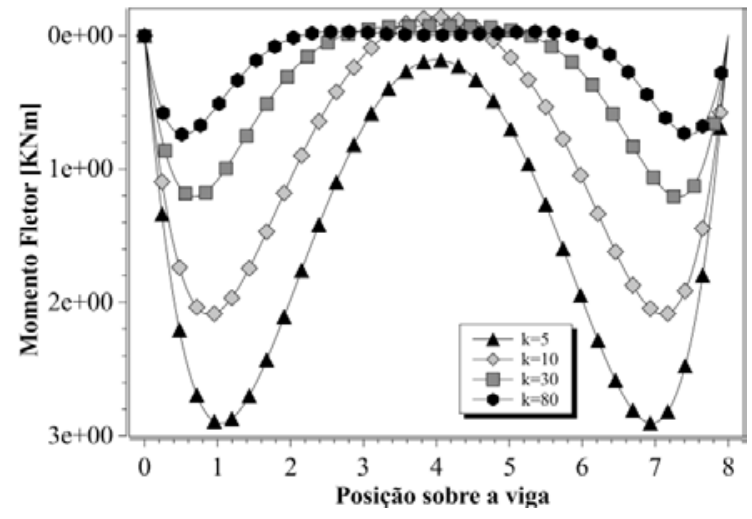

Fonte: Elaborado pelo autor, (2020).

Verifica-se na Figura 8 como o diagrama de momento fletor é influenciado pelo fator $k$. Isto evidencia que, para solos com baixa rigidez, o esforço interno da viga é maior, necessitando de uma análise cuidadosa no dimensionamento para resistir a tais solicitações. A Figura 9 representa a variação do deslocamento com a redução do coeficiente de recalque até a nulidade. 
Figura 9: Influência do fator $k$.

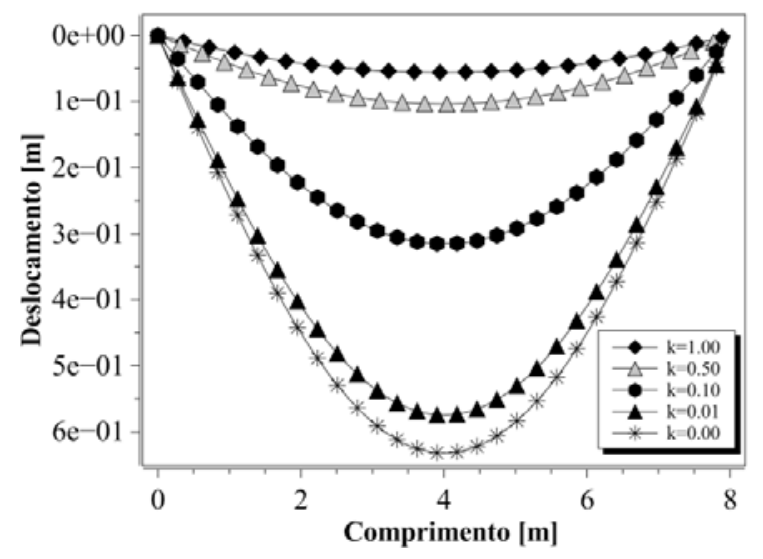

Fonte: Elaborado pelo autor, (2020).

Uma análise sobre a curva acima revela que, quanto menor o fator $k$, o perfil de deslocamento tende para o caso com $k=0$, que, por sua vez, representa o caso de uma viga sem contato com o solo $\left(k_{v} w=0\right)$, respeitando a solução clássica de viga. Dessa maneira, o segundo caso é uma viga biapoiada na qual atua uma carga concentrada cujo módulo é igual $100 \mathrm{kN}$ e que está localizada no meio do vão da viga, de comprimento $L=8 \mathrm{~m}$, conforme mostrado na Fig.(10). Considera-se o coeficiente de recalque com valor de $80 \mathrm{MNm}^{-3}$ e com seção transversal de $20 x 15 \mathrm{~cm}$.

Figura 10: Viga biapoiada com carregamento concentrado.

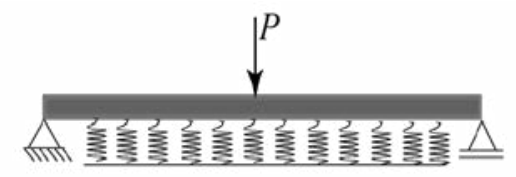

Fonte: Elaborado pelo autor, (2020).

De forma análoga ao primeiro caso, a validação é direcionada pela comparação com a solução analítica do problema específico, vide Hetényi (1971). A Figura 11 expõe a curva de erro médio com o aumento do número de pontos no domínio discreto.

Figura 11: Erro médio percentual para viga com carga concentrada.

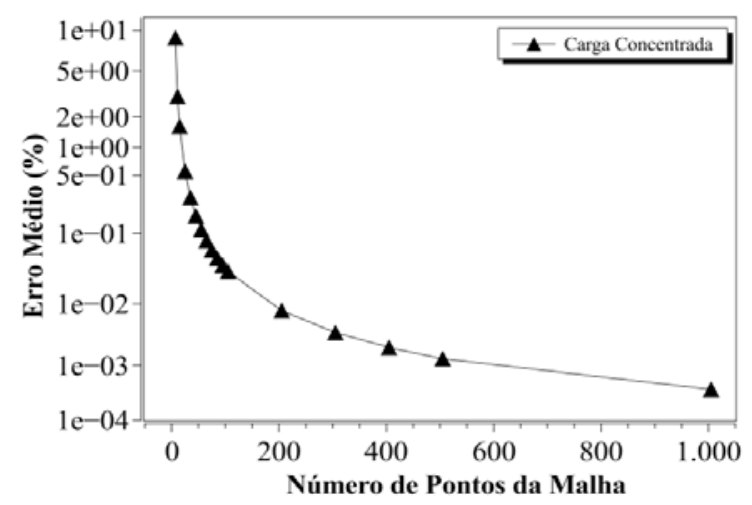

Fonte: Elaborado pelo autor, (2020). 
O comportamento da curva evidencia uma queda contínua com o refinamento da malha, com erro em torno de $10^{-4}$. Esse fato confirma o bom desempenho do método no presente teste. Com o problema devidamente validado, é possível explorar as particularidades do caso. A Figura 12 apresenta o deslocamento da viga, correlacionando os valores analíticos e numéricos obtidos pelo método das diferenças finitas.

Figura 12: Campo de deslocamento transversal da viga.

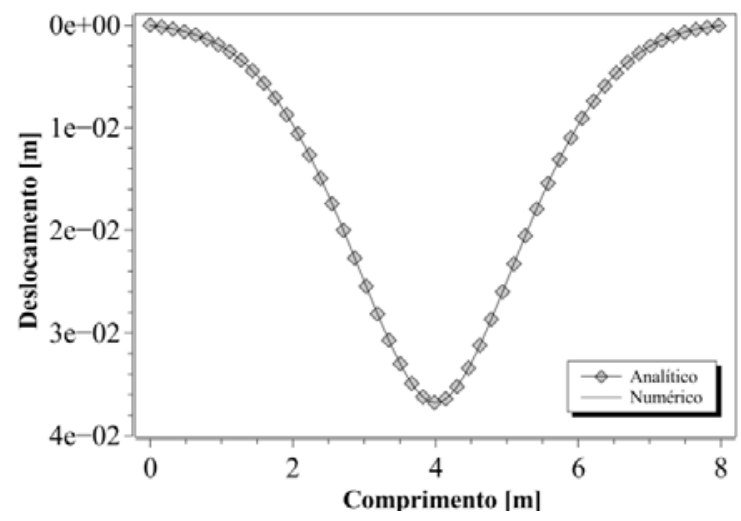

Fonte: Elaborado pelo autor, (2020).

Devido à natureza do carregamento e à vinculação, é fácil de notar que os pontos da malha próximos aos apoios são aproximadamente nulos, em detrimento ao deslocamento no meio do vão da viga, em quese torna máximo. Entre as diversas aplicações e usos das técnicas numéricas, a determinação dos diagramas de esforços internos através do campo de deslocamento se torna uma tarefa fácil e rápida. A Figura 13 expõe o gráfico do momento fletor da viga.

Figura 13: Diagrama de momento fletor.

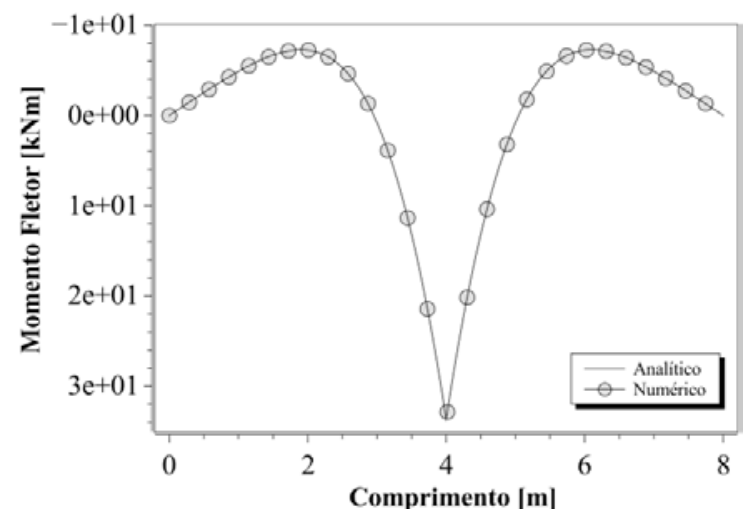

Fonte: Elaborado pelo autor, (2020).

Pela ilustração acima, fica clara a simetria do diagrama de momento fletor, quando atua uma carga pontual no meio da viga. Nota-se que a solução numérica está alinhada com a solução analítica do problema. Na Figura 14 apresenta-se um gráfico de esforço de cortante. 
Figura 14: Diagrama de esforço cortante.

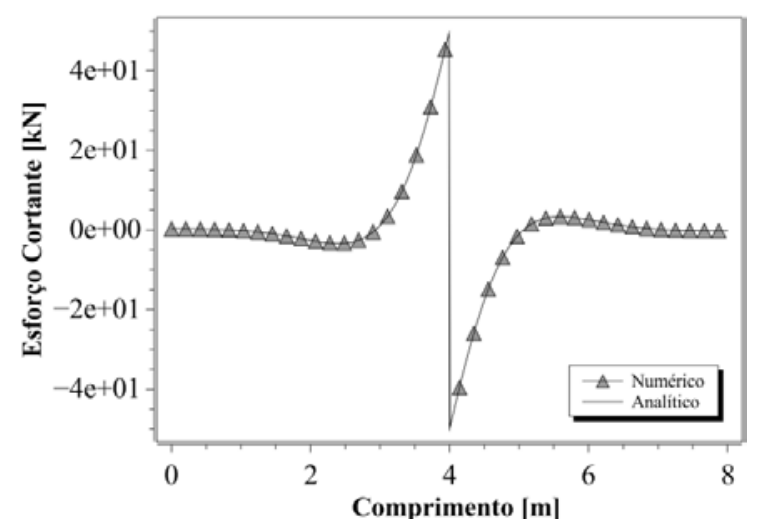

Fonte: Elaborado pelo autor, (2020).

O diagrama de esforço cortante apresenta um comportamento particular, isto é, existe uma descontinuidade no meio do vão da viga. Para explorar com detalhe tal fato, a Figura 15 esboça o erro da solução do esforço cortante próximo ao meio do vão da viga.

Figura 15: Intervalo de erro ao redor do meio do vão da viga.

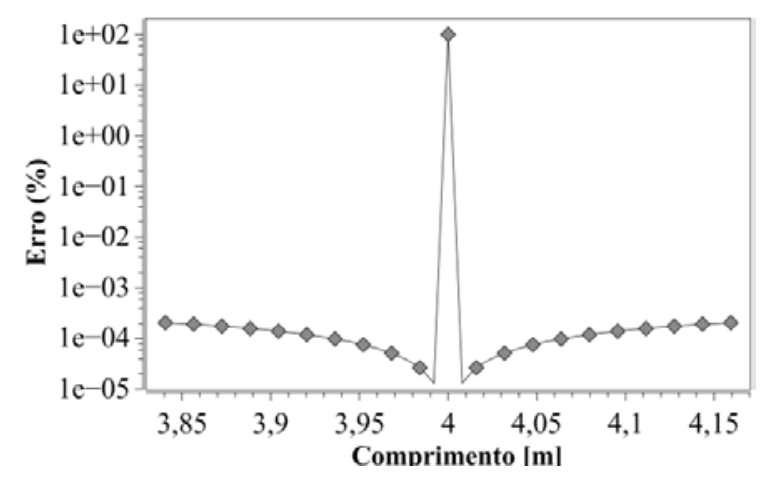

Fonte: Elaborado pelo autor, (2020).

A partir de uma rápida análise da Figura 15, constata-se que, no ponto central, o erro tem salto devido à descontinuidade. Porém, para malhas bem refinadas, os pontos vizinhos em relação ao ponto central apresentam bons resultados, contornando o problema. Ressalta-se que, para métodos numéricos com natureza integral, como elementos finitos e contorno, tal problema de descontinuidade possui pouca influência na aplicação.

A exposição gráfica dos diagramas de esforço internos e deslocamento são fundamentais em projetos e detalhamentos de cálculo estrutural. Contudo, no contexto prático da engenharia, os dimensionamentos são direcionados através dos valores máximos das grandezas, a fim de garantir a resistência da peça solicitada em projeto. Desse modo, a Tabela 2 apresenta uma análise paramétrica da rigidez do solo em relação aos momentos e deslocamentos máximos. 
Tabela 2 - Relação entre $k$ e os momentos e recalque do solo.

\begin{tabular}{cccc}
\hline$k\left[\mathrm{MNm}^{-3}\right]$ & $\begin{array}{c}\text { Recalque } \\
\text { máximo }[\mathrm{mm}]\end{array}$ & $\begin{array}{c}\text { Momento Positivo } \\
\text { Máximo }[\mathrm{kNm}]\end{array}$ & $\begin{array}{c}\text { Momento Negativo } \\
\text { Máximo }[\mathrm{kNm}]\end{array}$ \\
\hline 5 & 36,778 & 33,645 & $-7,285$ \\
10 & 21,879 & 28,490 & $-6,090$ \\
30 & 9,624 & 21,655 & $-4,493$ \\
60 & 5,722 & 18,205 & $-3,783$ \\
80 & 4,611 & 16,942 & $-3,522$ \\
100 & 3,901 & 16,023 & $-3,331$ \\
150 & 2,878 & 14,478 & $-3,010$ \\
200 & 2,319 & 13,473 & $-2,801$ \\
250 & 1,962 & 12,742 & $-2,649$ \\
\hline
\end{tabular}

Fonte: Elaborado pelo autor, (2020).

Na Figura 16, pode-se avaliar a influência do coeficiente de recalque na redução dos momentos máximos positivos e negativos e o deslocamento da viga.

Figura 16: Influência do fator $k$.

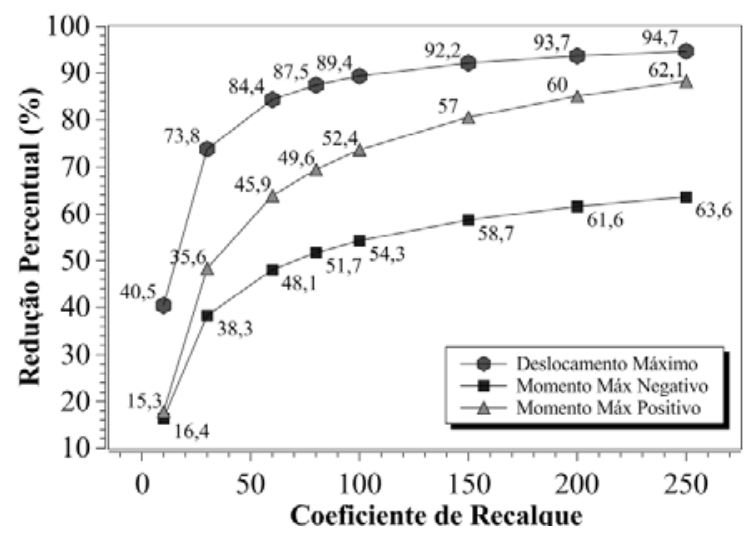

Fonte: Elaborado pelo autor, (2020).

Para quantificar os valores das grandezas, tomou-se como referência o solo com $k=5 \mathrm{MNm}^{-3}$. Para um solo com rigidez alta houve uma redução no deslocamento em quase $95 \%$. Em relação aos momentos máximos da viga, ocorre uma redução parelha entre ambos: com o aumento da rigidez do solo chega-se a um percentual de aproximadamente $63 \%$. Por fim, o terceiro caso é uma viga biapoiada com comprimento de $L=8 m$ submetida a três tipos de carregamentos, conforme Figura 17.

Figura 17: Viga biapoiada com múltiplos carregamentos.

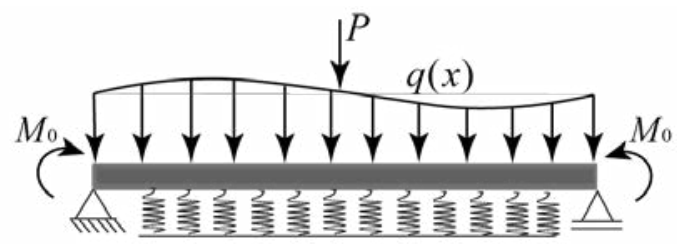

Fonte: Elaborado pelo autor, (2020). 
Esse caso é escolhido para verificar a generalidade do programa computacional desenvolvido para casos de carregamentos diversos. O carregamento distribuído é uma carga senoidal com o formato posto pela Equação, em que $q_{0}=80 \mathrm{kNm}^{-1}$ e $m=5$.

$$
q(x)=q_{0} \sin \left(\frac{m \pi x}{L}\right)
$$

A força concentrada tem intensidade de $100 \mathrm{kN}$ e o momento concentrado nas bordas da viga com módulo igual a $50 \mathrm{kNm}$. Com intuito de validar os resultados, usam-se as soluções analíticas presentes em Hetényi (1971), Jones (1997) e Vlasov (1966). A Figura 18 apresenta a curva de erro médio percentual.

Figura 18: Erro médio percentual para viga com múltiplos carregamentos.

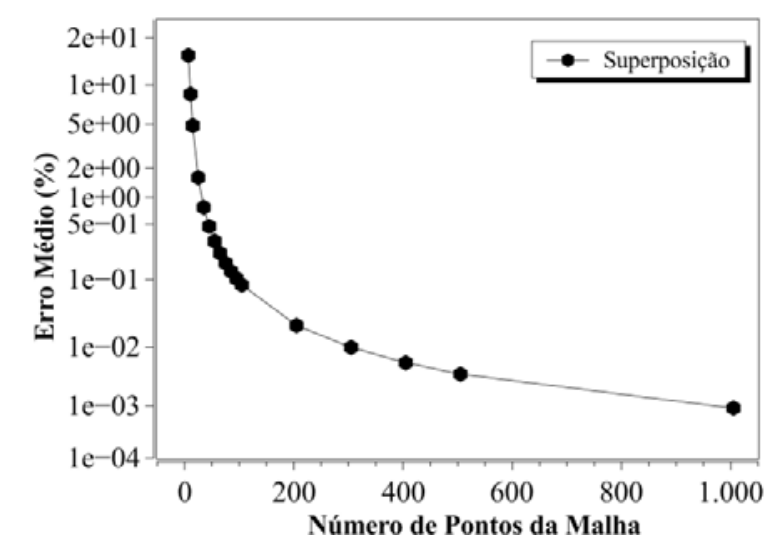

Fonte: Elaborado pelo autor, (2020).

Numa análise da curva de validação, mostrada na Figura 18, fica visível o comportamento decrescente do erro de forma monotônica. Esse fato evidencia o bom desempenho do método nesse problema. Em particular ao terceiro caso, a solução analítica não é direta como os casos vistos anteriormente. Dessa maneira, se faz necessário o uso do princípio da superposição de efeitos para desenvolver uma solução analítica para tal problema, como mostrado pela Eq. :

$$
w(x)=w_{q}+w_{P}+w_{M_{0}}
$$

Em que:

$$
\begin{aligned}
& w_{q}=\text { deslocamento pela carga distribuída senoidal }(\mathrm{m}) \\
& w_{p}=\text { deslocamento pela carga concentrada no meio do vão }(\mathrm{m}) ; \\
& w_{M 0}=\text { deslocamento ação dos momentos concentrados nas extremidades }(\mathrm{m}) ;
\end{aligned}
$$

A solução expressa acima é constituída pela soma das soluções dos casos da viga sujeita a uma carga senoidal, submetida à força pontual no meio do vão, e para uma viga com momentos concentrados nas bordas. Ressalta-se que, em casos com carregamentos complexos, a solução analítica se torna uma tarefa cansativa e nem sempre trivial. Nesse cenário, o uso de métodos numéricos torna-se essencial, dinamizando todo o processo de solução. A modelagem de vigas sob uma base elástica envolve dois parâmetros essenciais interligados com a interação solo-estrutura. A priori, o primeiro parâmetro está ligado à rigidez da viga, como mostra a Figura 19, que expõe o efeito da variação da seção transversal. Para tanto, considera-se um solo com coeficiente de recalque de $80 \mathrm{MNm}^{-3}$. 
Figura 19: Análise paramétrica da rigidez da viga.

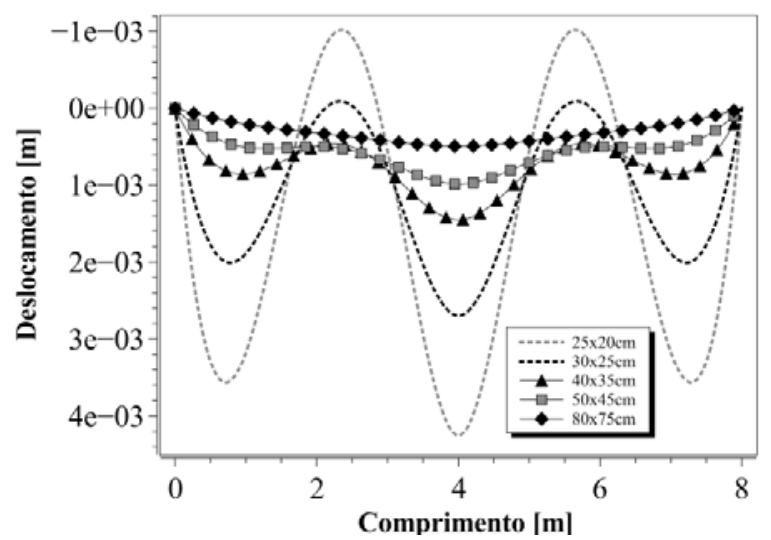

Fonte: Elaborado pelo autor, (2020).

Assume-se que o módulo de elasticidade é constante, o que induz que a rigidez da viga varia somente com características geométricas. Dessa maneira, o aumento da seção transversal proporciona uma maior rigidez à flexão da viga. Observa-se, na Figura 19, que o campo de deslocamento possui uma menor deformação ao longo do comprimento da viga. Em contrapartida, quando a rigidez da viga é menor, o campo de deslocamento é afetado significativamente, tanto pelo efeito da carga externa como pela reação do solo.

Na prática, a viga é um elemento que se ajusta conforme a necessidade em projeto, contudo o solo não tem essa característica, variando suas propriedades de acordo com a região estudada. Assim, fixa-se a seção transversal da viga em $20 \times 15 \mathrm{~cm}$.

Figura 20: Análise paramétrica da rigidez do solo.

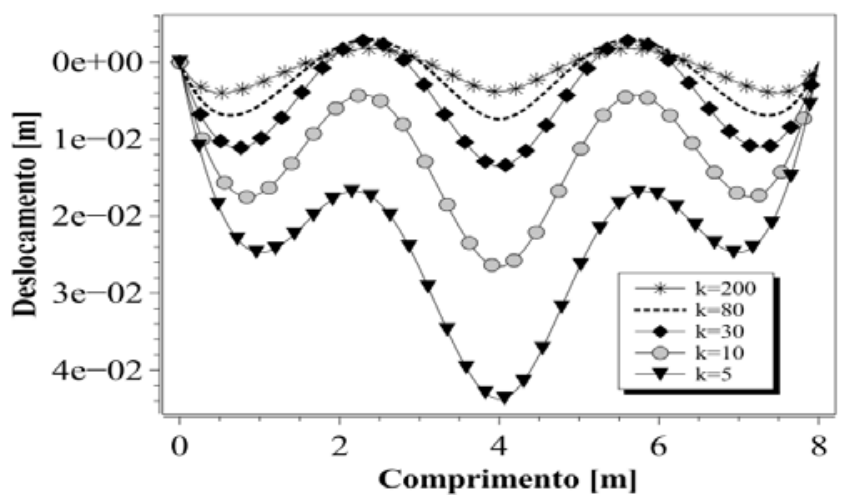

Fonte: Elaborado pelo autor, (2020).

Verifica-se, ao analisar a Figura 20, que o deslocamento da viga varia com o fator $k$, assim, em solos com menor resistência, o efeito da carga externa torna-se mais predominante. Porém, em solos com maior resistência, o perfil do deslocamento sofre uma influência significativa do solo.

\section{Conclusão}

Em síntese, a experimentação numérica conduzida pelo método das diferenças finitas apresentou um bom desempenho na solução dos problemas propostos, e erros percentuais baixos quando comparados à ordem de grandeza. Dessa maneira, fica evidente que, para as aplicações estudadas, o método mostra-se uma ferramenta eficaz e versátil para análises preliminares em vigas de fundação.

Para o papel de ensino-aprendizagem, o método das diferenças finitas surge como uma ferramenta potencializadora para o docente em sala de aula, fornecendo uma alternativa motivacional aos alunos, 
possibilitando um entendimento natural sobre os conceitos relativos aos métodos numéricos. Esse método torna-se uma excelente estratégia para ser introduzida no escopo de uma disciplina sobre introdução às técnicas aproximadas ou estudos preliminares sobre simulação computacional.

Logo, é possível criar um forte elo entre o binômio discente-docente através da transmissão de conceitos correlacionados a todo entendimento físico acerca do processo de modelagem e simplificações previstas nas formulações matemáticas do problema físico em questão. Atrelado a esse âmbito, é possível explorar os conceitos básicos e abstratos adquiridos ao longo do ciclo de ensino, como cálculo, física, programação, álgebra etc., agora concatenados e exigidos nas técnicas numéricas. Portanto, a exploração e as iniciativas para o uso de métodos numéricos são imprescindíveis na seara da educação, servindo como caminho de motivação para a pesquisa cientifica em cursos de graduação.

\section{Referências}

ALVES DE SOUZA, R.; HEMETÉRIO CORDEIRO DOS REIS, J. Interação solo-estrutura para edifícios sobre fundações rasas. Acta Scientiarum. Technology, [S.I.], v. 30, n. 2, p. 161-171, 20 out. 2008.

ANTONIAZZI, J. P. Interação solo-estrutura de edifícios com fundações superficiais. Orientador: Gerson Moacyr Sisniegas Alva. 2011. 138f. Dissertação (Mestrado em Engenharia Civi) - Santa Maria, Universidade Federal De Santa Maria, 2011.

ARISTIZÁBAL-OCHOA, J. D. Estructuras de vigas sobre suelos elásticos de rigidez variable. Revista Internacional de Desastres Naturales, Accidentes e Infraestructura Civil, [S.I.], v. 3, n. 2, p. 157-174, 2003.

BOWLES, J. E. Analytical and computer methods in foundation engineering. [S.I.]: McGraw-Hill Companies, 1974.

CUNHA, M. C. C. Métodos numéricos. São Paulo: Editora da UNICAMP, 2000.

HACHICH, W. C. et al. Fundações: teoria e prática. São Paulo: Pini, 1998.

HETÉNYI, M. Beams on elastic foundation: theory with applications in the fields of civil and mechanical engineering. [S.I.]: University of Michigan, 1971.

JONES, G. Analysis of beams on elastic foundations: using finite difference theory. New York: Thomas Telford, 1997.

NIKODÝM, M.; FRYDRÝŠEK, K. Finite difference method applied for the beams on elastic foundationexamples. In: MECHANICAL STRUCTURES AND FOUNDATION ENGINEERING, 2010. Proceedings [...]. [S.I.:S.n.], 2010.

PAVAN, R. C.; COSTELLA, M. F.; GUARNIERI, G. Soil-structure interaction for frame structures on shallow foundations. Revista IBRACON de Estruturas e Materiais, [S. I.], v. 7, n. 2, p. 273-285, 2014.

RIBEIRO, D. B. Análise da interação solo-estrutura via acoplamento MEC-MEF. Orientador: João Batista de Paiva, 2005. 119f. Dissertação (Mestrado em Engenharia de Estruturas) - Escola de Engenharia de São Carlos, Universidade de São Paulo, São Carlos, 2005.

TANAHASHI, H. Formulas for an infinitely long Bernoulli-Euler beam on the Pasternak model. Soils and Foundations, [S.I.], v. 44, n. 5, p. 109-118, 2004.

TEODORU, I. Analysis of beams on elastic foundation: the finite differences approach. In: Technical Conference for Doctoral Study, 9., 2007 [S.I.], Proceedings, [S.I.]: Brno University of Technology, 2007.

TIMOSHENKO, S. Resistência dos materiais. Rio de Janeiro: Ao Livro Técnico, 1969. 
VLASOV, V. Z. Beams, plates and shells on elastic foundations. Jerusalém: Israel Program for Scientific Translations, 1966

WANG, Y. H.; THAM, L. G.; CHEUNG, Y. K. Beams and plates on elastic foundations: a review. Progress In: Structural Engineering and Materials, [S.I.], v. 7, n. 4, p. 174-182, 2005.

\section{Sobre os autores}

Natan Sian das Neves

Doutorando em Engenharia civil pela Universidade Federal do Rio de Janeiro, (2020). Mestre em Engenharia civil, área de estruturas, pela Universidade Federal do Espírito Santo, (2019). Graduado em Engenharia civil pela Faculdade Capixaba da Serra (Multivix /Serra), 2017.

Avaliado em: 23.03 .2020

Aceito em: 06.04.2020 